\title{
Enhanced Spatial Resolution During Locomotion and Heightened Attention in Mouse Primary Visual Cortex
}

\author{
Patrick J. Mineault, ${ }^{1}$ Elaine Tring, ${ }^{1}$ Joshua T. Trachtenberg, ${ }^{1}$ and $\odot$ Dario L. Ringach ${ }^{1,2}$ \\ ${ }^{1}$ Department of Neurobiology, David Geffen School of Medicine, University of California, Los Angeles, California 90095, and ${ }^{2}$ Department of Psychology, \\ University of California, Los Angeles, California 90095
}

We do not fully understand how behavioral state modulates the processing and transmission of sensory signals. Here, we studied the cortical representation of the retinal image in mice that spontaneously switched between a state of rest and a constricted pupil, and one of active locomotion and a dilated pupil, indicative of heightened attention. We measured the selectivity of neurons in primary visual cortex for orientation and spatial frequency, as well as their response gain, in these two behavioral states. Consistent with prior studies, we found that preferred orientation and spatial frequency remained invariant across states, whereas response gain increased during locomotion relative to rest. Surprisingly, relative gain, defined as the ratio between the gain during locomotion and the gain during rest, was not uniform across the population. Cells tuned to high spatial frequencies showed larger relative gain compared with those tuned to lower spatial frequencies. The preferential enhancement of high-spatial-frequency information was also reflected in our ability to decode the stimulus from population activity. Finally, we show that changes in gain originate from shifts in the operating point of neurons along a spiking nonlinearity as a function of behavioral state. Differences in the relative gain experienced by neurons with high and low spatial frequencies are due to corresponding differences in how these cells shift their operating points between behavioral states.

Key words: alertness; locomotion; neuronal gain; operating point; spatial acuity; visual cortex

Significance Statement

How behavioral state modulates the processing and transmission of sensory signals remains poorly understood. Here, we show that the mean firing rate and neuronal gain increase during locomotion as a result in a shift of the operating point of neurons. We define relative gain as the ratio between the gain of neurons during locomotion and rest. Interestingly, relative gain is higher in cells with preferences for higher spatial frequencies than those with low-spatial-frequency selectivity. This means that, during a state of locomotion and heightened attention, the population activity in primary visual cortex can support better spatial acuity, a phenomenon that parallels the improved spatial resolution observed in human subjects during the allocation of spatial attention.

\section{Introduction}

Efficient sensory representations must adapt continually to behavioral demands, allocating limited resources to the processing of relevant sensory stimuli (Lennie, 2003; Harris and Thiele, 2011; Lee and Dan, 2012). Even in the awake state, levels of attention and alertness fluctuate over time (Reimer et al., 2014). In

Received Feb. 8, 2016; revised April 18, 2016; accepted May 4, 2016.

Author contributions: P.J.M., J.T.T., and D.L.R. designed research; P.J.M., E.T., and D.L.R. performed research; P.J.M. and D.L.R. analyzed data; P.J.M., J.T.T., and D.L.R. wrote the paper.

This work was supported by the National Institutes of Health (Grant EY-023871 to J.T.T. and Grant EY-018322 to D.L.R.). We thank Robert Shapley for valuable discussions and comments on a previous version of this manuscript, Pablo Garcia Junco-Clemente and Nick Olivas for experimental support, and anonymous reviewers for detailed comments and critiques.

The authors declare no competing financial interests.

Correspondence should be addressed to Dario L. Ringach, Department of Psychology, UCLA, 502 Portola Plaza, Los Angeles, CA 90095-1563. E-mail: dario@ucla.edu.

DOI:10.1523/JNEUROSCI.0430-16.2016

Copyright $\odot 2016$ the authors $\quad 0270-6474 / 16 / 366382-11 \$ 15.00 / 0$ mice, one can observe spontaneous transitions between states of rest, in which the mouse is still and the pupil is constricted, and one in which the mouse is actively moving and the pupil is dilated. In humans, fluctuations of pupil size correlate with changes in attention and cognitive load (Beatty, 1982). Moreover, changes in pupil size are correlated with activity in the locus ceruleus (Murphy et al., 2014), the sole source of norepinephrine to the cortex and one of key modulators of behavioral state (Sara, 2009). These links suggest that the mouse may provide a simple model to study how cortical processing is modulated by fluctuating levels of attention. Indeed, a number of recent studies have explored this possibility (Poulet and Petersen, 2008; Goard and Dan, 2009; Bereshpolova et al., 2011; Harris and Thiele, 2011; Bennett et al., 2013; Polack et al., 2013; Arroyo et al., 2014; Reimer et al., 2014; Zhuang et al., 2014).

Here, we continue this line of research by testing whether a salient phenomenon observed in human visual attention studies has a counterpart in the mouse visual system. A feature of spatial 
attention in humans is that it increases spatial resolution, as measured by the improvement of performance in acuity, texture segmentation, and visual search tasks at the attended location (Carrasco et al., 2002; Carrasco and Yeshurun, 2009). Spatial attention, triggered by cueing, is also known to improve acuity in nonhuman primates (Golla et al., 2004). It has been suggested, based on the outcomes of various psychophysical studies (Carrasco and Barbot, 2014), that the neural mechanism responsible for the improvement in spatial resolution is a preferential increase in the gain of neurons with high preferred spatial frequencies (Anton-Erxleben and Carrasco, 2013). Interestingly, in mice, prior work has shown that visually evoked responses increase during locomotion relative to rest, with tuning curves being modulated multiplicatively (Niell and Stryker, 2010). However, it is unknown whether changes in gain are uniform across the entire cortical population or if they depend on their tuning properties. Here, we set out to replicate the observation that gain increases during locomotion and to determine whether these changes depend on the preferred spatial frequency of the neurons in a way that would support enhanced spatial resolution during locomotion.

There is increasing evidence that, in addition to a simple modulation of neuronal gain, primary visual cortex (V1) participates in a more complex processing of multimodal signals. These include the combination of visual and running speeds within single cells (Saleem et al., 2013), increases in spatial summation of visual neurons during locomotion compared with rest (Ayaz et al., 2013), the detection of a mismatch between predicted and actual visual input as a function of locomotion (Keller et al., 2012), and the presence of reward timing signals (Shuler and Bear, 2006). Here, we focus on how the cortical representation of spatial frequency varies between rest and locomotion by adopting experimental methods that kept these other factors constant in our measurements. Our goal was to determine whether changes in behavioral state affect the low-level representation of the retinal image in V1.

\section{Materials and Methods}

Animals

All procedures were approved by University of California-Los Angeles's Office of Animal Research Oversight (the Institutional Animal Care and Use Committee) and were in accord with guidelines set by the National Institutes of Health. A total of 30 C57BL/6J mice (Jackson Laboratory), both male (10) and female (20) at postnatal day 35 (P35)-P56 of age were used in this study. Mice were housed in groups of two to three in a reversed light cycle. Animals were naive subjects with no prior history of participation in research studies. A total of 129 different fields were imaged and data were obtained data for 7018 cells, for a median of 47 cells per field (range: 6-162).

\section{Surgery}

Carprofen and buprenorphine analgesia were administered preoperatively. Mice were then anesthetized with isoflurane $(4-5 \%$ induction; $1.5-2 \%$ surgery). Core body temperature was maintained at $37.5^{\circ} \mathrm{C}$ using a feedback heating system. Eyes were coated with a thin layer of ophthalmic ointment to prevent desiccation. Anesthetized mice were mounted in a stereotaxic apparatus. Blunt ear bars were placed in the external auditory meatus to immobilize the head. A portion of the scalp overlying the two hemispheres of the cortex $(\sim 8 \mathrm{~mm}$ by $6 \mathrm{~mm})$ was removed to expose the underlying skull. After the skull was exposed, it was dried and covered by a thin layer of Vetbond. After the Vetbond dried ( $\sim 15 \mathrm{~min})$, it provided a stable and solid surface on which to affix an aluminum bracket with dental acrylic. The bracket was affixed to the skull and the margins sealed with Vetbond and dental acrylic to prevent infections.

\section{Virus injection}

A 3-mm-diameter region of skull overlying the occipital cortex was removed. Care was taken to leave the dura intact. GCaMP6-fast (UPenn Vector Core: AAV1.Syn.GCaMP6f.WPRE.SV40; \#AV-1-PV2822) was expressed in cortical neurons using adeno-associated virus (AAV). AAVGCaMP6-fast (titer: $\sim 4 \times 10^{13}$ genomes $/ \mathrm{ml}$ ) was loaded into a glass micropipette and slowly inserted into the V1 using a micromanipulator. Two injection sites were made near the center of V1 separated $\sim 200 \mu \mathrm{m}$ apart. For each site, AAV-GCaMP6-fast was pressure injected using a Picospritzer III (Parker) (4 puffs at 15-20 pounds per square inch with a duration of $10 \mathrm{~ms}$, each puff separated by $4 \mathrm{~s}$ ) starting at a depth of 350 $\mu \mathrm{m}$ below the pial surface and making injections every $10 \mu \mathrm{m}$ moving up, with the last injection made at $100 \mu \mathrm{m}$ below the pial surface. The total volume injected across all depths was $\sim 0.5 \mu$ l. The injections were made automatically by a computer program in control of the micromanipulator and the Picospritzer.

A sterile, 3-mm-diameter cover glass was then placed directly on the dura and sealed at its edges with VetBond. When dry, the edges of the cover glass were further sealed with dental acrylic. At the end of the surgery, all exposed skull and wound margins were sealed with VetBond and dental acrylic. Mice were then removed from the stereotaxic apparatus, given a subcutaneous bolus of warm sterile saline, and allowed to recover on the heating pad. When fully alert, they were placed back in their home cages.

\section{Imaging}

Once expression of GCaMP6f was observed in V1, typically between 11 and $15 \mathrm{~d}$ after the injection, imaging sessions took place. Imaging was performed using a resonant, two-photon microscope (Neurolabware) controlled by Scanbox acquisition software. The light source was a Coherent Chameleon Ultra II laser running at $920 \mathrm{~nm}$. The objective was an $\times 16$ water-immersion lens (Nikon, 0.8 numerical aperture, 3 mm working distance). The microscope frame rate was $15.6 \mathrm{~Hz}$ (512 lines with a resonant mirror at $8 \mathrm{kHz}$ ). Eye movements and pupil size were recorded via a Genie M1280 camera (Teledyne Dalsa) fitted with a $740 \mathrm{~nm}$ longpass filter that looked at the eye indirectly through the reflection of an infrared-reflecting glass (see Fig. 1A). Eye velocity was computed as changes of the pupil center in the image per frame of the microscope. Images were captured at an average depth of $210 \mu \mathrm{m}$ (90\% of imaging fields within the range $80-320 \mu \mathrm{m}$ ). During imaging, a substantial amount of light exits the brain through the pupil. Therefore, no additional illumination was required to image the pupil. Mice were free to walk on a platform that was mounted on a rotary, optical encoder (US Digital) connected to an Arduino Mega 2560 board, which provided direct access to movement information. Both locomotion and eye movement data were synchronized to the microscope frames.

\section{Visual stimulation}

Hartley stimuli (Ringach et al., 1997; Malone and Ringach, 2008) were generated in real time by a processing sketch using OpenGL shaders (see http://processing.org). The stimulus was updated 4 times/s on a BenQ XL2720Z screen refreshed at $60 \mathrm{~Hz}$. The screen measured $60 \mathrm{~cm}$ by $34 \mathrm{~cm}$ and was viewed at a $20 \mathrm{~cm}$ distance, thereby subtending $112 \times 80$ degrees of visual angle. The maximum spatial frequency was 0.15 cycles $/{ }^{\circ}$, which corresponds to 12 cycles along the vertical extent of the display. The Hartley set consisted of the following gratings:

$$
H_{k_{x}, k_{y}}= \pm \operatorname{cas}\left(2 \pi \frac{k_{x} x+k_{y} y}{M}\right)
$$

where $\operatorname{cas}(x) \equiv \cos (x)+\sin (x)$ and the wavenumbers $k_{\mathrm{x}}$ and $k_{\mathrm{y}}$ represent the number of cycles along the horizontal and vertical axes, respectively, and these indices ran between -12 and 12, excluding the origin $\left(k_{x}, k_{y}\right)=(0,0)$. Therefore, the total number of different images in the Hartley set was $\left((2 \times 12+1)^{2}-1\right) \times 2=1248$. The responses to the four spatial phases that are present at each combination of orientation and spatial frequency were averaged, leading to 312 locations in the orientation and spatial frequency domain. Each combination was presented, on average, 15.4 times during a 20 -minute-long stimulus refreshed at $4 \mathrm{~Hz}$. This stimulus update rate was selected in an attempt to 
collect data as fast as possible while still evoking reliable responses from cells.

A transistor-transistor logic pulse was generated by an Arduino board at each stimulus update transition. The pulse was sampled by the microscope and time stamped with the frame and line number being scanned at that time. The time stamps provided a way to align the visual stimulation and imaging data in time.

The screen was calibrated using a Photo-Research PR-650 spectroradiometer and the result used to generate the appropriate gamma corrections for the red, green, and blue components via an nVidia Quadro K4000 graphics card. The contrast of the stimulus was $99 \%$. The center of the monitor was positioned with the center of the receptive field population for the eye contralateral to the cortical hemisphere under consideration. The location of the receptive fields were estimated by an automated process in which localized, flickering checkerboard patches appeared at randomized locations within the screen. This experiment was run at the beginning of each imaging session to ensure the centering of receptive fields on the screen.

\section{Data processing}

Motion stabilization. Calcium images were aligned to correct for motion artifacts in a two-step process. First, images were aligned rigidly in a recursive fashion to correct for slow drifts in the imaging plane. Pairs of neighboring images in time were aligned by finding the peak of their cross-correlation and then pairs of averages of such pairs were aligned and so on. In the second step, images were aligned nonrigidly to a reference mean image to correct for fast in-plane movements, which are frequently observed during grooming. The Lucas-Kanade algorithm was applied iteratively (Greenberg and Kerr, 2009) to match a reference mean image nonrigidly, refining the estimate of this reference mean image after each alignment iteration.

Segmentation. After motion stabilization, a MATLAB (The MathWorks) graphical user interface tool developed in our laboratory was used to define regions of interest corresponding to putative cell bodies manually. Correlation and kurtosis images were used to identify cell candidates (Smith and Häusser, 2010). The correlation image, corresponding to the average correlation of a pixel and its eight neighbors across time, highlighted regions of space that covary in time. The kurtosis image highlights regions in space with signals composed of large, infrequent deviations: putative spikes. These images were computed after subtracting linear trends (Pnevmatikakis et al., 2014).

These images were used to identify approximately circular regions of space of an appropriate radius visually with high correlation and high kurtosis. Clicking a seed pixel at the center of such a candidate patch allowed the definition of a region of interest by flood filling an image corresponding to the correlation of the highlighted pixel and every other pixel in the image field (Ozden et al., 2008). The interface then allowed the user to grow or shrink the region of interest dynamically to a desired size.

Signal extraction and spike inference. After segmentation, signals were extracted by computing the mean of the calcium fluorescence within each region of interest. Non-negative deconvolution (Vogelstein et al., 2010; Pnevmatikakis et al., 2014) was used to estimate spikes from calcium traces. The inverse, constrained form of the non-negative deconvolution problem was solved (Pnevmatikakis et al., 2014) using the CVX package (Boyd and Vandenberghe, 2004). To mitigate the effect of drifting background fluorescence, the offset was modeled as slowly moving in time with a 10-knot cubic spline. The noise of the measured calcium signals was estimated as the median absolute deviation of the first-order derivative divided by a factor of $.6745 \sqrt{2} \approx .954$ (Johnstone and Silverman, 1997).

The constrained deconvolution method of Pnevmatikakis et al. (2014) requires the specification of the impulse response of the calcium indicator. An exponential impulse response function was assumed and its decay time estimated using reference data consisting of simultaneous loose-seal cell-attached recordings and calcium imaging of GCaMP6f in visual neurons (Chen et al., 2013). This dataset was resampled at a sampling rate of $15.5 \mathrm{~Hz}$, non-negative deconvolution was run for a grid of values of decay times, the $R^{2}$ of the estimated calcium signal and the ground-truth cell spike trains across the 11 cells of the dataset were calculated, and the parameter set with the largest mean $R^{2}$ was selected. This yielded a decay time $\tau_{1 / 2}=135 \mathrm{~ms}$, for a validated mean $R^{2}$ of 0.42 (cf. supplemental Table 3 in Chen et al., 2013). Our analyses and the resulting interpretation assume that there is an approximately linear relationship between the inferred spike rate and the actual spike rates from the neurons, as partly justified by existing data (see Fig. 3E in Chen et al., 2013).

\section{Generic linear model}

The response of a neuron to a stimulus was assumed to be given by the following linear model:

$$
y(t)=\sum_{\omega_{x}, \omega_{y}, \tau} s\left(\omega_{x}, \omega_{y}, t-\tau\right) w\left(\omega_{x}, \omega_{y}\right) v(\tau)+\operatorname{ar}(t)+b+\epsilon(t)
$$

where $\epsilon(t)$ is independent, identically distributed Gaussian noise, $s\left(\omega_{x}, \omega_{y}, t\right)$ is the stimulus presented at time $t, w\left(\omega_{x}, \omega_{y}\right)$ is the Fourier kernel, $v(\tau)$ is the temporal kernel, $b$ is the offset during rest, $a$ is the change in offset during locomotion, $y(t)$ is the measured response, and $r(t)$ is an indicator variable taking the value 1 when the instantaneous velocity of the animal is at least $1 \mathrm{~cm} / \mathrm{s}$ and zero otherwise. The parameter $a$ in the equation allows for shifts in the baseline as a function of state.

This model was fit through alternating least-squares (Ahrens et al., 2008) and the norm of the temporal kernel was constrained to 1. A smoothness penalty was used for the spatial kernel (Wu et al., 2006) and its strength was determined by fivefold cross-validation.

The quality of fit of this generic linear model was compared with a baseline model as follows:

$$
y(t)=\operatorname{ar}(t)+b+\epsilon(t)
$$

A fit was considered significant whenever the cross-validated sum-ofsquared error $S S E_{\mathrm{L}}$ of the generic linear model was such that:

$$
r_{L}=\sqrt{1-S S E_{L} / S S E_{B}} \geq 0.15
$$

where $S S E_{B}$ corresponds to the sum-of-squared error of the baseline model and $r_{L}$ is analogous to a correlation (Pearson's $r$ ) value. A total of $3803 / 7018$ (54.2\%) neurons were significantly tuned according to this criterion.

\section{Parametric linear model}

To estimate the preferred spatial frequency and orientation, as well as their respective bandwidth, a parametric linear model was fit as follows:

$$
\begin{array}{r}
y(t)=\sum_{\omega_{x,} \omega_{y}, \tau} s\left(\omega_{x}, \omega_{y}, t-\tau\right) w\left(\omega_{x}, \omega_{y} \mid r, \theta, \sigma_{r}, A_{\theta}\right) v(\tau)+\operatorname{ar}(t)+b \\
+\epsilon(t) w\left(\omega_{x}, \omega_{y} \mid r, \theta, \sigma_{r}, A_{\theta}\right)=\exp \left(A_{\theta} \cos \left(\arctan \left(\omega_{y} / \omega_{x}\right)-\theta\right)\right. \\
\left.-\left(\log \left(1+\sqrt{\omega_{x}^{2}+\omega_{y}^{2}}\right)-\log (1+r)\right)^{2} / 2 / \sigma_{r}^{2}\right)
\end{array}
$$

where the Fourier kernel is a separable function in orientation and spatial frequency. The orientation tuning is a von Mises function and the spatial frequency tuning is a log-Gaussian function. The parameter $r=\sqrt{\omega_{x}^{2}+\omega_{y}^{2}}$ represents the optimal spatial frequency and $\theta=\arctan \left(\omega_{y} / \omega_{x}\right)$ the optimal orientation. The parameter $A_{\theta}$ controls the sharpness of tuning in the orientation domain and $\sigma_{r}$ controls the sharpness of tuning in spatial frequency. This model was fit through alternating nonlinear least-squares using the solution to the generic linear model as a seed for the temporal kernel and an initial grid search to find optimal values for $r, \theta, \sigma_{r}, A_{\theta}$. A total of 3476/7018 (49.5\%) neurons were considered significant $(r>0.15)$.

\section{Separable linear models}

In some analyses, the gain of the linear model was quantified as a function of state by fitting the linear model as follows:

$$
\begin{array}{r}
y(t)=\sum_{\omega_{x}, \omega_{y}, \tau}(\operatorname{cr}(t)+d) s\left(\omega_{x}, \omega_{y}, t-\tau\right) w\left(\omega_{x}, \omega_{y}\right) v(\tau)+\operatorname{ar}(t) \\
+b+\epsilon(t)
\end{array}
$$


where $\operatorname{cr}(t)+d$ correspond to the state-dependent gain. The gain during locomotion is then given by $c+d$ and the gain during rest is $d$. The relative gain change from rest to locomotion, from the model parameters, is given by $1+c / d$. The relative gain is analogous to the concept of an attentional field (Reynolds and Heeger, 2009). The model was fit through alternating least-squares and declared significant if it attained a cross-validated $r$-value of 0.15 attained by $3861 / 7018$ (55.0\%) of neurons.

\section{Inseparable linear models}

In one instance (see Fig. 2D,E), spatial and temporal kernels were allowed to depend on whether the animal was in locomotion or rest. Therefore, the generic and parametric linear models of the previous sections were fit contingent on whether the animal was undergoing locomotion (criterion: instantaneous velocity of $1 \mathrm{~cm} / \mathrm{s}$ ). The factor $\operatorname{ar}(t)$ was ignored in this case. Fits were declared significant if the model could achieve a cross-validated correlation value of at least 0.15 in both the rest and locomotion conditions in 2605/7018 (37.1\%) neurons.

\section{Bayesian decoder}

Poisson independent decoder. To examine how locomotion influences the representation of stimuli, the framework of Jazayeri and Movshon (2006) was followed. A brief overview of the framework is provided here. The goal is to decode the stimulus by estimating the probability that a population response was generated by a given stimulus $\theta_{j}$ out of a finite range of possibilities, $p\left(\theta=\theta_{j}\right)$. It was assumed that the $i$-th of $N$ neurons has a tuning curve $f_{i}(\theta)$ and that, on a given trial, $n_{i}$ spikes were observed from this neuron. Assuming that each neuron follows independent Poisson statistics and that each stimulus is equally likely, it follows from Bayes' theorem that:

$$
p\left(\theta=\theta_{j} \mid n_{i}\right) \propto \prod_{i} \frac{f_{i}\left(\theta_{0}\right)^{n_{i}} \exp \left(-f_{i}\left(\theta_{j}\right)\right)}{n_{i} !}
$$

Taking the log and removing the normalization constant, which is independent of $\theta$, the following is found:

$$
\begin{aligned}
L\left(\theta_{j}\right) & =\sum_{i} n_{i} \log \left(f_{i}\left(\theta_{j}\right)\right)-f_{i}\left(\theta_{j}\right) \\
& =\sum_{i} n_{i} \log \left(f_{i}\left(\theta_{j}\right)\right)+b\left(\theta_{j}\right)
\end{aligned}
$$

From this, the properly normalized distribution is recovered as follows:

$$
p\left(\theta=\theta_{j} \mid n_{i}\right)=\frac{\exp \left(L\left(\theta_{j}\right)\right)}{\sum_{k} \exp \left(L\left(\theta_{k}\right)\right)}
$$

The log-likelihood $L\left(\theta_{j}\right)$ can be computed straightforwardly as the sum of the log of tuning curves weighted by the number of spikes measured from each neuron, with an offset to compensate for biases in the population representation. It is possible to construct a two-layer neural network carrying equivalent information to $L\left(\theta_{j}\right)$ (Jazayeri and Movshon, 2006) and a point estimate corresponding to the maximum likelihood estimate of the stimulus can be obtained by a recurrent network (Deneve et al., 1999).

Homogeneous gain increase. The effect that a homogenous gain increase has on population decoding will now be discussed. The SD of a Poisson neuron is proportional to the square root of its mean; signal-tonoise ratio thus increases when the rate of a neuron increases. Intuitively, therefore, if a population of Poisson-like neurons increases their gain by a common factor in response to a change of state, then decoding should be facilitated.

Under the Poisson-independent decoder, a homogeneous gain of $\alpha$ has the effect of multiplying the log-likelihood (Eq. 8) by a factor $\alpha$. It follows from Equation 9 that, under an increase in gain, probabilities therefore become more extreme: $p\left(\theta=\theta_{j}\right)$ values that were most likely before the gain increase become still more likely after gain increase and values that were less likely become still less likely. Therefore, an increase in gain across the board decreases the uncertainty of the decoder and, because the decoder is unbiased, this implies that decoding error decreases when gain increases.

\section{Nuisance factor}

Now suppose, more generally, that neurons are modulated by a nuisance factor $\phi$ not relevant to decoding the parameter of interest; for example, locomotion, contrast, etc. Then, tuning curves become a function of both the parameter of interest and the nuisance factor:

$$
f_{i}(\theta, \phi)
$$

Assuming that the nuisance factor modulates tuning curves in an arbitrary fashion, then a different decoder must be used for every value of the nuisance parameter; equivalently, one decoder of the form in Equation 8, can be used, with synaptic weights modulated dynamically by the nuisance parameter. In either case, downstream decoding becomes more complicated. However, if modulation is multiplicative, that is, tuning curves are separable, then:

$$
f_{i}(\theta, \phi)=g_{i}(\theta) h_{i}(\phi)
$$

In this case $L\left(\theta_{j}\right)$ has a very simple form:

$$
\begin{aligned}
L\left(\theta_{j}\right) & =\sum_{i} n_{i} \log \left(f_{i}\left(\theta_{j}, \phi\right)\right)+b\left(\theta_{j}, \phi\right) \\
& =\sum_{i} n_{i} \log \left(g_{i}\left(\theta_{j}\right)\right)+n_{i} \log \left(h_{i}\left(\theta_{j}\right)\right)+b\left(\theta_{j}, \phi\right) \\
& =\sum_{i} n_{i} \log \left(g_{i}\left(\theta_{j}\right)\right)+b\left(\theta_{j}, \phi\right)+k
\end{aligned}
$$

where $k$ is independent of $\phi$. Equation 12 shows that, when the nuisance parameter modulates tuning curves multiplicatively, the stimulus can be decoded in exactly the same way as before except that the offset $b\left(\theta_{0}, \phi\right)$ now changes with $\phi$.

\section{Empirical decoder}

Graf et al. (2011) showed that the theoretical decoder of the previous section can underperform when the population has correlated noise or does not follow Poisson statistics exactly. Equation 12 can be relaxed to compensate for these deviations from the theoretical model. Factors corresponding to log tuning curves are replaced with empirically derived weights as follows:

$$
L\left(\theta_{j}\right)=\sum_{i} n_{i} W_{i j}+b_{j}(\phi)
$$

Absorbing the offset into a design matrix $\mathbf{x}$, this linear model for the likelihood can be rewritten into the canonical form:

$$
L(\boldsymbol{\theta}) \equiv \eta=\mathbf{x}^{T} \mathbf{W}
$$

As highlighted previously, this non-normalized log-likelihood can be transformed to a normalized likelihood by the softmax transformation:

$$
\boldsymbol{\mu}=\operatorname{softmax}(\boldsymbol{\eta})
$$

Finally, under this model, $\mu$ is the probability distribution of the categorical stimulus variable $y$ and it follows that it has a multinomial distribution:

$$
\mathbf{y} \sim \operatorname{Multinomial}(\boldsymbol{\mu})
$$

Therefore, this this empirical decoder can be interpreted as a special instance of multinomial regression, itself a special case of generalized linear models (McCullagh and Nelder, 1989).

\section{Decoder variants, fitting, and validation}

To measure how locomotion modulates decoding accuracy, both theoretical and empirical decoders were fit to the data.

In the case of the theoretical decoder, the number of spikes in a threeframe window centered on the mean temporal latency of all cells within an experiment were counted. The tuning curves smoothed by a Gaussian kernel were then computed to derive model weights. The size of the smoothing kernel was determined by minimizing the fivefold cross-validation decoding error. This decoding error was derived by selecting maximum likelihood (ML) estimates under the model and computing their root mean squared error (RMSE) relative to the actual stimuli in Fourier space.

For the empirical decoder, model weights were estimated by maximum a posteriori estimation through convex optimization in the frame- 
A

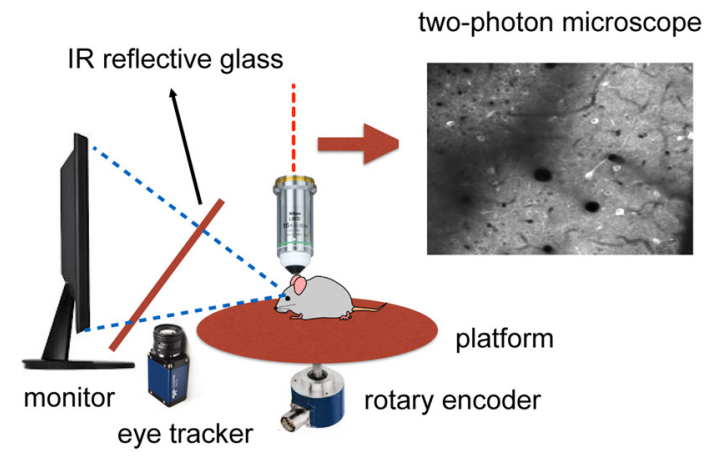

B
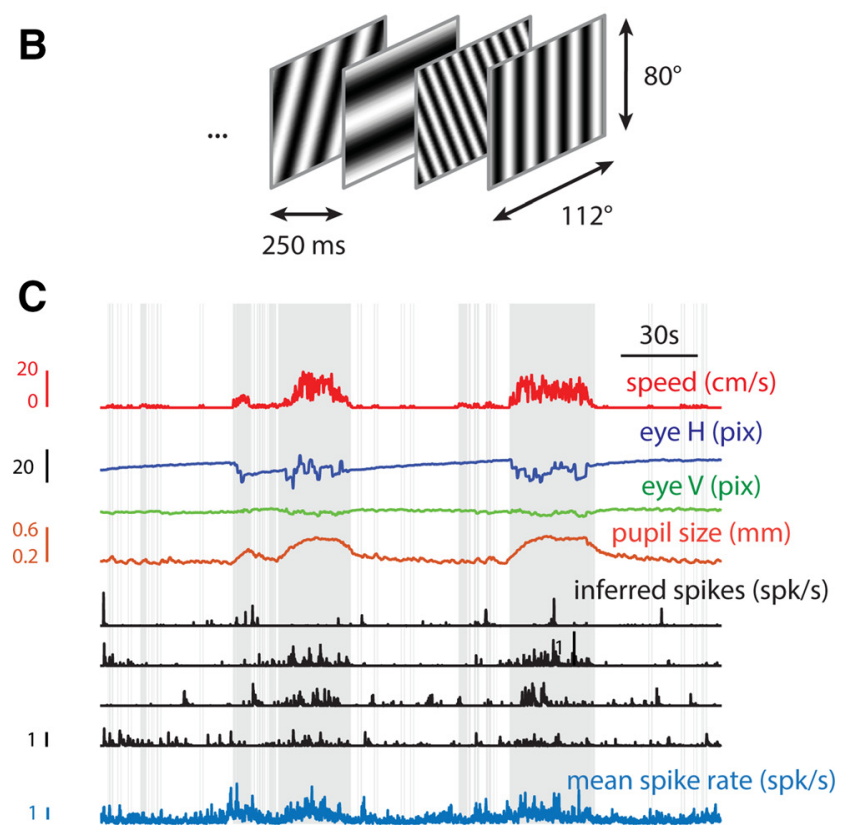

Figure 1. Experimental setup. $\boldsymbol{A}$, Activity of cells in V1 was imaged while a mouse, on a freely rotating platform, observed a continuous visual stimulus. The head was horizontal. $\boldsymbol{B}$, Sample of a visual stimulus sequence. The stimulus consisted of a pseudorandom sequence of gratings drawn from a Hartley basis set presented at a rate of 4 frames $/ s$. C, Segment of a data record depicting platform speed, eye position, pupil size, the inferred spikes of four sample cells, and the mean spike rate of the population. Gray bars in this and subsequent figures indicate periods of locomotion defined by a threshold on the speed signal.

work of generalized linear models (McCullagh and Nelder, 1989). Rather than directly counting spikes, the model was allowed to have a continuous temporal window, which was learned from the data using alternating optimization. A smoothness penalty on the log-tuning curves with strength determined by fivefold cross-validation was used. As with the theoretical decoder, the RMSE was computed for the maximum likelihood estimates. In addition, the RMSE for a Bayesian point estimate, which uses the whole estimated probability distribution, and not simply its maximum, was computed to minimize the expected RMSE.

Decoding was more accurate during locomotion for theoretical, empirical ML, and empirical Bayesian decoders; in absolute terms, the empirical Bayesian decoder outperformed the empirical ML, which outperformed the theoretical decoder. In Figure 5, the results of the more familiar empirical ML decoder are shown.

To verify that the improved stimulus representation could be used advantageously by a simple decoder in which behavioral state only modifies the prior, separate decoders were also fit for the rest and locomotion states using the same criteria as previous analyses. At prediction time, the prediction of the decoder relevant to the current state was used. Figure 5 shows that the decoding accuracy was very similar between these more complex, dual decoders and the single "efference copy" decoder, as measured by the empirical ML error. Similar results were obtained for the theoretical and empirical Bayesian decoders (data not shown).
Decoding accuracy as a function of spatial frequency

The same empirical decoders described in the previous section were used to examine the dependence of the decoding accuracy on spatial frequency and state (see Fig. 5). The ability of the model to predict the probability of the orientation of the stimulus given the absolute spatial frequency and spike trains was assessed as follows:

$$
p\left(\theta \mid \omega, n_{i}\right)
$$

However, the model actually defines:

$$
p\left(\omega_{x}, \omega_{y} \mid n_{i}\right)
$$

The probability distribution (Eq. 17) was approximated by considering a small range of spatial frequencies around the target spatial frequency:

$$
\begin{array}{r}
p\left(\theta=\arctan \left(\omega_{y}, \omega_{x}\right) \mid \omega, n_{i}\right) \\
\approx \frac{p\left(\omega_{x}, \omega_{y} \mid n_{i}\right) \mathrm{I}\left(\left|\sqrt{\omega_{x}^{2}+\omega_{y}^{2}}-\omega\right| \leq \alpha\right)}{\sum_{\omega_{x}, \omega_{y}} p\left(\omega_{x}, \omega_{y} \mid n_{i}\right) \mathrm{I}\left(\left|\sqrt{\omega_{x}^{2}+\omega_{y}^{2}}-\omega\right| \leq \alpha\right)}
\end{array}
$$

where $\alpha$ corresponds to the range of spatial frequencies around the one considered, which was set to 0.0125 cycles $^{\circ}$. Both ML and Bayesian variants of this model were considered; Bayesian variants performed better, but otherwise, the main quantitative effects (see Fig. 5) were similar in both variants. The Bayesian variant was used for the data shown in Figure 5.

\section{Motion onset analysis}

To measure the temporal relationship between motion onset, decoding accuracy, and firing rate, transitions between locomotion and rest were estimated as follows. An onset period was defined as one in which movement was slower than $0.7 \mathrm{~cm} / \mathrm{s}$ in $75 \%$ of time periods in the preceding $5 \mathrm{~s}$ and faster than $1 \mathrm{~cm} / \mathrm{s}$ in the $75 \%$ of time periods in the $5 \mathrm{~s}$ following. Onset times were defined as the centers of onset periods, discarding onset times separated by $<15 \mathrm{~s}$. Computed mean firing rate and RMSE around the time of locomotion onset were then computed. Offset times were defined similarly.

\section{Derivation of the gain of an exponential nonlinearity with a} normal input

Assume we have an exponential nonlinearity of the form $\mathrm{r}=A \exp (\lambda x)$, where the input is normally distributed $x \sim N(\mu, \sigma)$ (see Fig. 4B). Then, the slope of the best linear fit between the input and output was computed as: $g=(E\{r x\}-E\{r\} E\{x\}) / \sigma^{2}$. The terms in this equation can be calculated as follows:

$$
\begin{gathered}
E\{r x\}=E\{A \exp (\lambda x) x\}=A \exp \left(\lambda \mu+\lambda^{2} \mu^{2} / 2\right)\left(\mu+\lambda \sigma^{2}\right) \\
\bar{r}=E\{r\}=E\{A \exp (\lambda x)\}=A \exp \left(\lambda \mu+\lambda^{2} \mu^{2} / 2\right) \\
E\{x\}=\mu
\end{gathered}
$$

After substituting in the expression for the gain, the following is obtained:

$$
g=\lambda A \exp \left(\lambda \mu+\lambda^{2} \mu^{2} / 2\right)
$$

By using Equation 21, the expression can be seen to be equal to $g=\lambda \bar{r}$. In other words, the gain is proportional to the mean rate.

\section{Results}

To determine whether the representation of the image in V1 changes between locomotion and rest, we placed mice on a freely rotating platform and measured changes in the fluorescence of V1 pyramidal neurons expressing GCaMP6f (Chen et al., 2013) using two-photon laser scanning excitation (Fig. 1A). Mice were presented with a 20 -min-long sequence of high-contrast, sinusoi- 
A
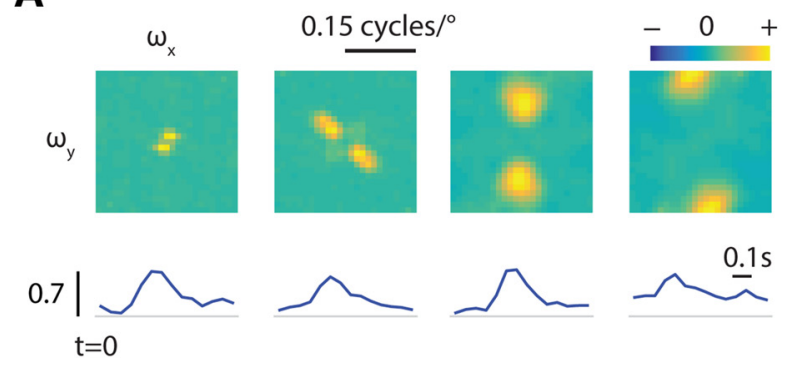

D

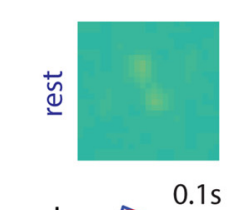

0.7

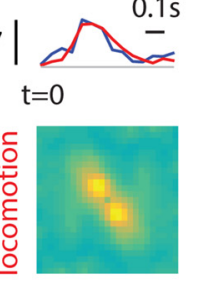

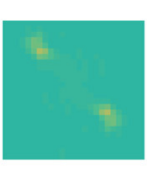
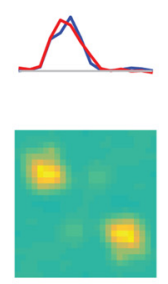
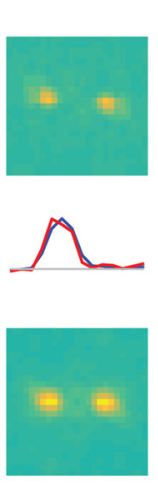
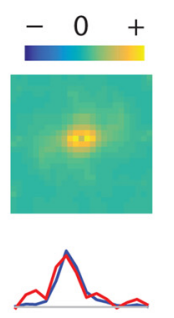

$\omega_{x}$

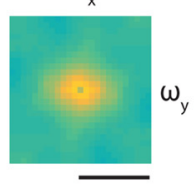

0.15 cycles $/^{\circ}$
B

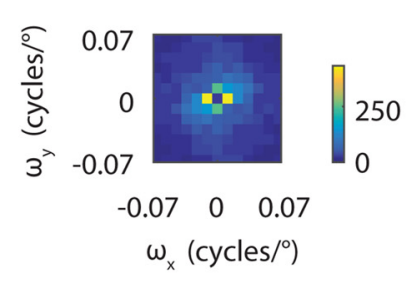

C $\quad \theta$
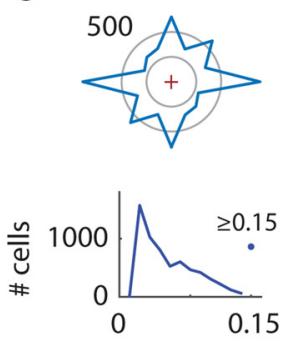

$\omega\left(\right.$ cycles $\left./^{\circ}\right)$
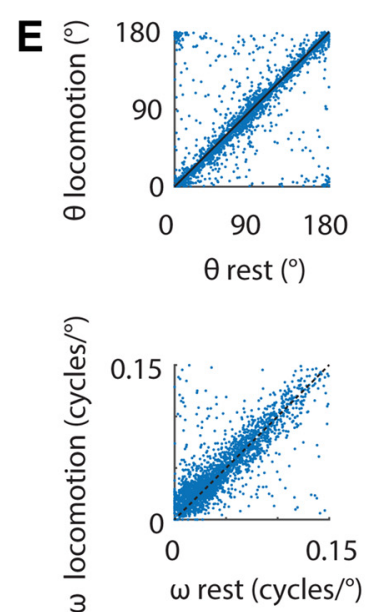

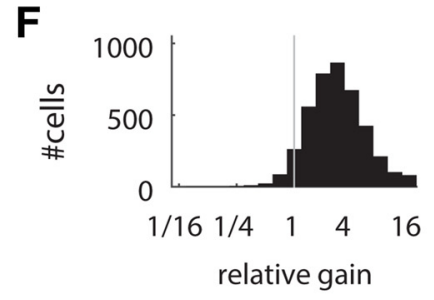

G

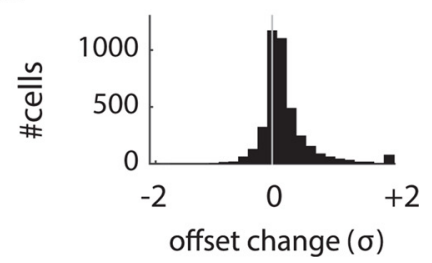

Figure2. Effect of behavioral state on the tuning of V1 neurons in the orientation and spatial-frequency (Fourier) plane. A, Tuning of four sample cells in the Fourier domain (origin is at the center) and their corresponding temporal kernels (bottom). These estimates were obtained by fitting a linear model to the data (Materials and Methods, Eq. 2). All temporal kernels are shown starting at $t=0$. Spatial frequency along the horizontal meridian is represented by $\omega_{\mathrm{x}}$ and the spatial frequency along the vertical meridian by $\omega_{\mathrm{y}}$. All Fourier kernels share the same pseudocolor scale. Positive values (red hues) represent stimuli with orientation and spatial frequency that led to increases in the response of the cell; negative values (blue hues) represent stimuli that suppressed the response of the cell; neutral stimuli are represented by a green hue. $\boldsymbol{B}$, Distribution of peak kernel positions in the Fourier domain across the population. The distribution is symmetric because the Fourier kernel is symmetric. C, Top, Distribution of preferred orientations, $\theta$. Bottom, Distribution of preferred spatial frequencies, $\omega=\sqrt{\omega_{x}^{2}+\omega_{y}^{2}}$. The symmetry of preferred orientations is a consequence of the symmetry of the Fourier kernels as well. Only well fit neurons (cross-validated $r>0.15, n=3476$; see Materials and Methods for details) are considered in this analysis. $\boldsymbol{D}$, Tuning of four sample cells in the Fourier domain during rest (top) and locomotion (bottom). Temporal responses are shown in the middle (blue: rest, red: locomotion). This was obtained by fitting a model that allowed changes in gain between locomotion and rest (Materials and Methods, Eq. 6). $\boldsymbol{E}$, Preferred spatial frequency and orientation are largely preserved across states. $\boldsymbol{F}$, Response gain increases substantially during locomotion. $\mathbf{G}$, There is a small but significant increase in baseline firing rate of $0.12 \pm 0.01$ SDs of the response during locomotion ( $p<0.001$, bootstrap test).

dal gratings that had random orientations and spatial frequencies (Hartley basis functions) refreshed at a rate of $4 \mathrm{~Hz}$ (Ringach et al., 1997; Malone and Ringach, 2008; Fig. 1B). During these sessions, mice spontaneously switched between periods of rest and locomotion (Fig. 1C). Such transitions were marked by correlated changes in platform velocity, the rate of horizontal eye saccades, pupil size, and mean population firing rate (Fig. 1C; Niell and Stryker, 2010; Polack et al., 2013; Erisken et al., 2014).

A continuous visual stimulation with constant mean luminance and contrast was selected to prevent external visual events from inducing changes in cortical state (Tan et al., 2014) while allowing us to map the tuning of cells. The relatively fast rate and unpredictability of the stimulus sequence helped keep sensory mismatch between the visual and motor signals at a constant level, likely alleviating its influence on V1 activity (Keller et al., 2012). Moreover, the stimulus sequence consisted of flashed gratings and contained no net motion, thereby minimizing the contribution of visual speed to the activity of neurons (Saleem et al., 2013).

From the responses of neurons to the Hartley sequence, we estimated the tuning of each cell in the joint spatial-frequency and orientation domain and their temporal responses via linear regression (Fig. 2A; Materials and Methods, Eq. 2). Many neu- rons were responsive to the stimulus sequence (defined as a crossvalidated $r>0.15$ in $3803 / 7018$ or $54.2 \%$ of the cells). To capture the preferences of neurons for orientation and spatial frequency, we fit a parametric model consisting of separable von Mises tuning for orientation and log-Gaussian spatial frequency tuning (Materials and Methods, Eq. 5). Although most neurons were tuned to low spatial frequencies, a large range of preferences was observed, as noted by the distribution of their preferred parameters in the Fourier plane (Fig. 2B,C). A few neurons preferred spatial frequencies close to or beyond the largest tested $(0.15$ cycles $/^{\circ}$; Fig. $2 C$, bottom).

To examine the effect of behavioral state on the tuning properties of individual neurons, we estimated receptive fields for periods of locomotion (defined by a platform speed $>1 \mathrm{~cm} / \mathrm{s}$ ) and rest (speed $<1 \mathrm{~cm} / \mathrm{s}$ ) separately. Temporal kernels were constrained to have unit norm in both locomotion and resting states. Therefore, any changes in gain were represented by changes in the amplitude of the Fourier kernels.

Consistent with prior studies, we found that locomotion led to increases in gain (Fig. 2D; Niell and Stryker, 2010; Bennett et al., 2013; Polack et al., 2013). We define the ratio between the gain during locomotion and the gain during rest as the "relative gain." Preferred orientation and spatial frequency were largely pre- 
served between locomotion and resting states (Fig. 2E). To investigate the behavior of the relative gain in more detail, we fit a model with a single tuning curve, but allowed for variations in baseline firing rate and gain across locomotion and rest states (see Materials and Methods, Eq. 6). We found that the relative gain across the population was $2.7 \pm 0.1$ (Fig. $2 F$, geometric mean, $p<0.001$, bootstrap test). There was a small but significant increase in baseline firing rate of $0.12 \pm 0.01$ SDs of the response (Fig. $2 G, p<0.001$, bootstrap test).

Next, we investigated whether the relative gain was dependent on the preferred stimulus parameters of the neurons. Indeed, we found that relative gain was largest for cells preferring high spatial frequencies (Fig. 3A). The relative gain for neurons preferring high spatial frequencies $\left(\omega>0.075\right.$ cycles $\left.^{\circ}, n=784\right)$ was $3.1 \pm 0.15$ (mean $\pm 95 \%$ confidence interval), which was higher than for cells preferring medium $(0.025<\omega \leq 0.075$ cycles $\left.^{\circ}, 2.7 \pm 0.1, n=654\right)$ and low spatial frequencies $\left(0<\omega<0.025\right.$ cycles $^{\circ}$, $2.45 \pm 0.08, n=388)$. In other words, neurons with high preferred spatial frequencies experienced relative gains $\sim 26 \%$ larger than cells tuned to the low spatial frequencies. A scatter plot of the same data captures the variability in the population (Fig. 3B). Even though this may appear to be a modest difference at first sight, we show below that this phenomenon has a marked effect on the ability of the entire population to encode high spatial-frequency information.

Although preferred spatial frequency was the major factor related to relative gain increase, we also observed a weaker modulation of relative gain with respect to preferred orientation (Fig. $3 C$ ). Curiously, the data hinted at larger relative gains at oblique orientations. To investigate whether there was a relationship between this trend and eye movements during the experiments, we computed the distribution of eye velocities, which showed a clear bias toward the horizontal (Fig. 3D), consistent with prior studies (Bennett et al., 2013). If an increase in horizontal retinal motion led to higher responses during locomotion, we would expect relative gain to be maximal in cells tuned to vertical orientations. However, there was no obvious peak at that location (Fig. $3 C$ ). Its absence suggests that eye movements and the resulting retinal slip are not a major contributor to increases in relative gain. In addition, we verified there are no major changes in eye torsion between rest and locomotion (Movies 1 and 2). An alternative explanation for this peculiar trend is offered below.

An important observation, which led us to propose a possible mechanism for the observed changes in gain, was the existence of an approximately linear relationship between gain and mean firing rates of neurons (Fig. 4A). This relationship arises if one postulates an exponential, spiking nonlinearity $r=A \exp (\lambda x)$, linking the response of the cell $r$ to a generator potential $x$ (Fig. 4B; Granit, 1955; Nykamp and Ringach, 2002; Ringach and Ma-
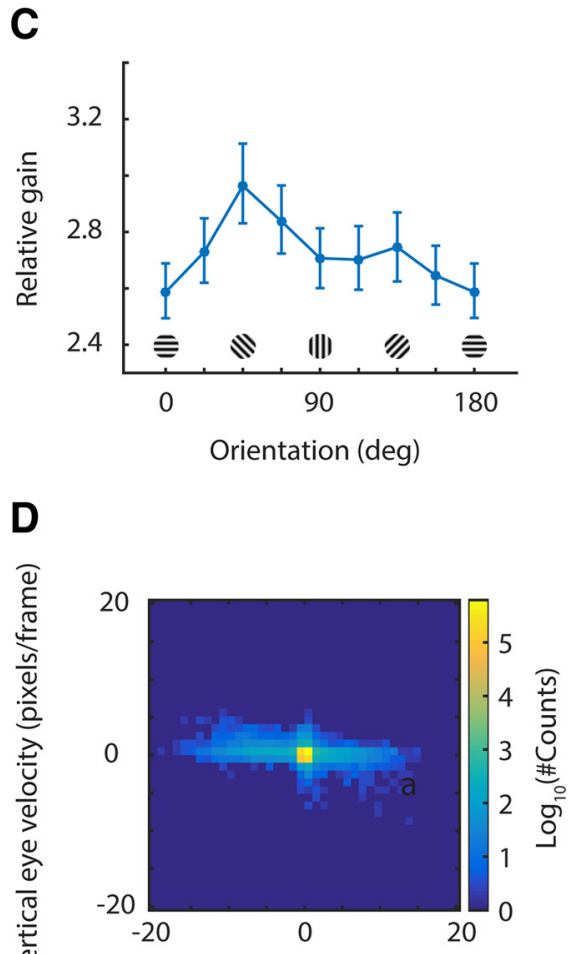

Horizontal eye velocity (pixels/frame)

Figure 3. Relative gain depends on preferred spatial frequency and orientation. $A$, Relative gain increases with preferred spatial in a log scale). Although most of the time the eyes are still, when they move, they tend to do so along the horizontal axis. The frequency of such movements is higher during locomotion (Fig. 10).

lone, 2007). If we assume the generator potential is normally distributed with mean $\mu$ and SD $\sigma$, then the mean spike rate is given by:

$$
\bar{r}=E\{A \exp (\lambda x) \mid x \sim N(\mu, \sigma)\}=A \exp \left(\lambda \mu+\lambda^{2} \sigma^{2} / 2\right)
$$

Moreover, it can be shown that the gain, which corresponds to the linear regression coefficient between the response and the generator potential, is simply $g=\lambda \bar{r}$ (see derivation in Materials and Methods). In other words, the gain is proportional to the mean response, consistent with the experimental observation (Fig. 4A).

If changes in gain reflect changes in the operating point of the cells between locomotion and rest (Bennett et al., 2013; Polack et al., 2013), then this simple model predicts that the relative gain across two states must be equal to the relative change in mean response, $g_{\text {loc }} / g_{\text {rest }}=\bar{r}_{l o c} / \bar{r}_{\text {rest }}$. Where $g_{\{l o c, \text { rest }\}}$ represents the gain during locomotion and rest, respectively, and we use a similar notation for the mean spike rates, $\bar{r}_{\{l o c, \text { rest }\}}$. Remarkably, this relationship holds reasonably well in our data $\left(r=0.62, p<10^{-10}\right.$; Fig. 4C).

In addition, if shifts in the operating points along a spiking nonlinearity explain changes in gain, it is possible that the difference in relative gain shown by neurons with high and low spatial frequency preferences is due to their operating points shifting in different ways. First, we find that cells with high spatial frequency preference have lower spiking rates than cells 


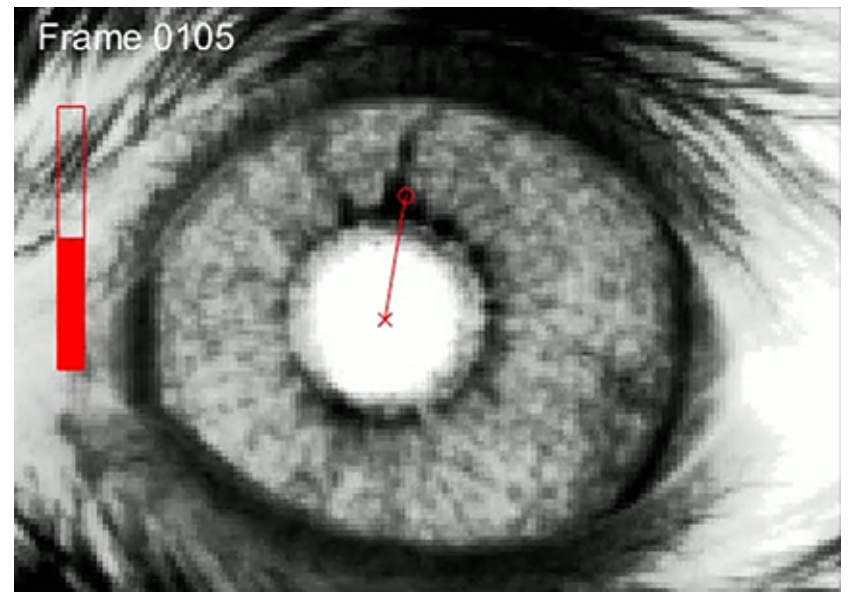

Movie 1. Example of the measurement of pupil size and torsional eye movements during locomotion. The red bar represents normalized, instantaneous running speed. Torsion is measured as the angle formed by the line joining the center of the pupil and a selected twodimensional feature of the iris and the horizontal axis in the image plane. While the pupil dilates during locomotion, torsion remains approximately constant.

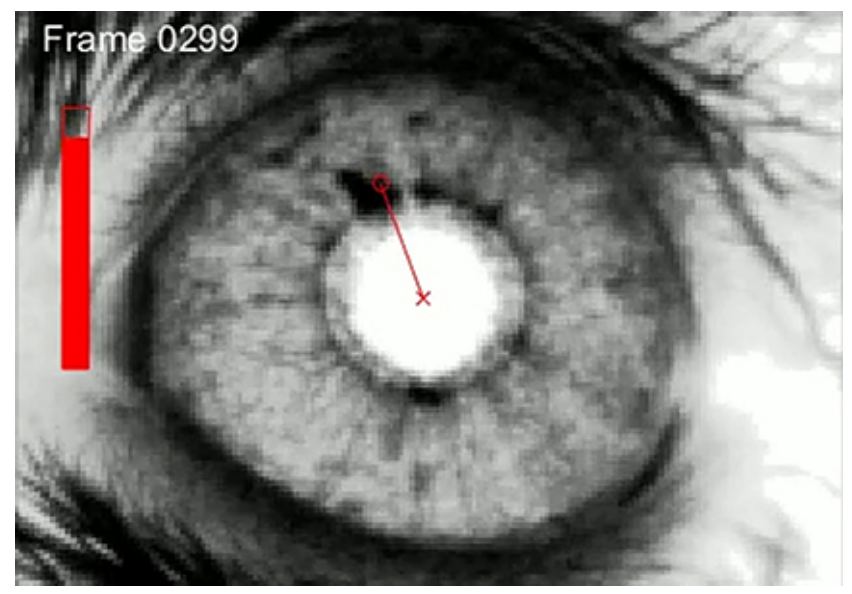

Movie 2. Another example of changes in pupil size and torsion during locomotion. The information is presented in the same format as in Movie 1.
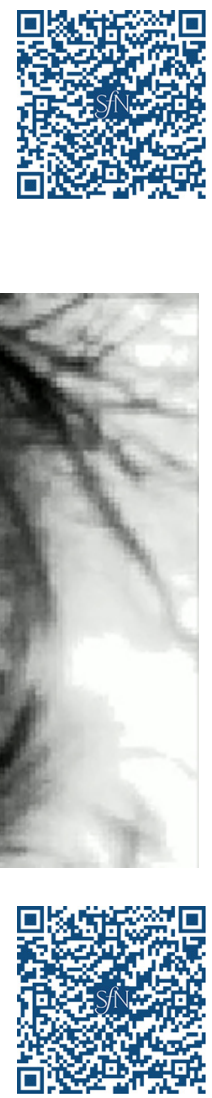

with low spatial frequency preference during both rest and locomotion (Fig. 4D). Second, increases in absolute firing rates between rest and locomotion experienced by these two groups are statistically indistinguishable, $\Delta \bar{r}_{\text {low }} \approx \Delta \bar{r}_{\text {high }}$ $=\Delta \bar{r}$ (rank-sum test, $p>0.32$; Fig. $4 D)$.

A graphic summary of these relationships helps to explain how the differential changes in gain between rest and locomotion may arise from the different operating points of the neurons (Fig. $4 E)$. In the figure, $\bar{r}_{\{l o w, h i g h\}}^{\text {rest }, \text { loc }\}}$ represent the mean rates of the two cell groups during rest and locomotion, and $\Delta \bar{r}$ is the increase in firing rate in the two groups between rest and locomotion. Then, according to the model:

$$
\frac{g_{\text {low }}^{\text {loc }}}{g_{\text {low }}^{\text {rest }}}=\frac{\bar{r}_{\text {low }}^{\text {loc }}}{\bar{r}_{\text {low }}^{\text {rest }}}=\frac{\bar{r}_{\text {low }}^{\text {rest }}+\Delta \bar{r}}{\bar{r}_{\text {low }}^{\text {rest }}}=1+\frac{\Delta \bar{r}}{\bar{r}_{\text {low }}^{\text {rest }}}
$$

and a similar relationship holds for the group of cells with high spatial frequency preference:

$$
\frac{g_{\text {high }}^{\text {loc }}}{g_{\text {high }}^{\text {rest }}}=1+\frac{\Delta \bar{r}}{\bar{r}_{\text {high }}^{\text {rest }}}
$$

However, the data show that the operating points during rest differ, such that $\bar{r}_{\text {rest }}^{\text {low }}>\bar{r}_{\text {rest }}^{\text {high }}$, implying that:

$$
\frac{g_{\text {low }}^{\text {loc }}}{g_{\text {low }}^{\text {rest }}}=1+\frac{\Delta \bar{r}}{\bar{r}_{\text {low }}^{\text {rest }}}<1+\frac{\Delta \bar{r}}{\bar{r}_{\text {high }}^{\text {rest }}}=\frac{g_{\text {high }}^{\text {loc }}}{g_{\text {high }}^{\text {rest }}}
$$

In other words, the relative gain for cells with low spatial frequency preference is smaller than the group with high spatial frequency preference.

Armed with these observations, we return to the puzzling dependence of relative gain changes with orientation (Fig. $3 B$ ). If our proposed explanation is correct, then we should expect to see a matching dependence of the operating point with orientation preference as well. Indeed, we see a tendency for cells with preferred orientations around vertical and horizontal to show larger firing rates during rest than cells tuned to the oblique orientations (Fig. 4F). Such trends can cause cells tuned for vertical and horizontal to experience smaller increases in gain than cells tuned to the oblique orientations, which is what is observed in the data (Fig. 3B). Altogether, these analyses indicate that the change in gain experienced by a neuron is tightly linked to the shift in its operating point between behavioral states, which, in turn, depends on its preferred spatial frequency and orientation.

Is the change in gain during locomotion sufficient to have an effect on the representation of visual information in V1? As gain (and consequently the signal-to-noise ratio of individual cells) increases during locomotion, the ability of an ideal observer to predict the stimuli that triggered a population response is expected to improve as well (see Materials and Methods). Moreover, if changes in state only modify the gain of individual cells while leaving their tuning invariant, the stimulus can be decoded in a manner where the behavioral state (rest or locomotion) only modifies the prior probability of stimuli (see Materials and Methods).

To verify this prediction using the collected data, we trained a Bayesian decoder to estimate the visual input from population activity (Graf et al., 2011; Fig. 5A). The decoder computed a spike-weighted sum of decoding fields to yield an estimate of the probability that the population response was generated by a given stimulus. The resulting distribution led to an estimate of a stimulus in the Fourier domain, from which we assessed the decoding error (Fig. 5B).

Population activity represented stimuli much more accurately during locomotion than during rest. The decoding error was strongly reduced during periods of locomotion relative to rest (Fig. 5C). Decoding error decreased rapidly after the onset of locomotion and increased more gradually before a transition to rest, indicating that the modulation was strongly locked to changes in mean spike rate and behavioral state (Fig. 5D). Importantly, using two separate decoders during locomotion and rest did not improve prediction errors (Fig. 5E). This indicates that the population can be appropriately modeled by a fixed set of kernels that change solely in gain and that the change approximately behavioral state is adequately captured by changes in the prior distribution (see Materials and Methods).

Finally, we determined whether the preferential enhancement found for cells tuned to high spatial frequencies could support enhanced spatial resolution in the V1 population. We repeated the decoding analysis, focusing on orientation decoding as a 
function of behavioral state and spatial frequency. Across all spatial frequencies, orientation was decoded more accurately during locomotion (Fig. 5F). However, the improvement relative to rest, using chance performance as the baseline, was more pronounced at high spatial frequencies (Fig. 5G).

\section{Discussion}

Previous research has shown that evoked firing rates increase and the detectability of weak stimuli improve during locomotion compared with rest (Niell and Stryker, 2010; Bennett et al., 2013; Polack et al., 2013). Our analyses indicate that individual cells modulate their gain in a way consistent with a shift in their operating point along an exponential nonlinearity. This explains the approximately linear relationship between gain and mean response rate (Fig. 4). An exponential nonlinearity was chosen because it is amenable to mathematical treatment and facilitates the explanation of how shifts in operating point modulate gain. Other accelerating nonlinearities, such as halfsquaring, can also generate an approximate linear relationship between gain and mean rate over an adequate range of inputs. To study these issues in more detail, it would be important to measure the dependence between the operating point and preferred stimulus parameters, as well as the shape of spiking nonlinearities, using intracellular recording techniques (Bennett et al., 2013; Polack et al., 2013; Tan et al., 2014).

The cortical circuit involved in modulating the operating point remains to be explored. Growing evidence indicates that, during periods of locomotion, VIP interneurons (cells expressing the vasoactive intestinal polypeptide) increase their firing rates and inhibit SOM interneurons (somatostatin-expressing inhibitory neurons; Fu et al., 2014). SOM-expressing cells have been implicated as the source of surround suppression in pyramidal neurons (PYR; Adesnik et al., 2012). The disinhibition resulting from the activation of the $V I P \rightarrow S O M \rightarrow P Y R$ circuit would explain the increase in spatial summation (Ayaz et al., 2013), the shift of operating points toward more depolarized states during locomotion compared with rest (Bennett et al., 2013; Polack et al., 2013), and the resulting increase in gain during locomotion.

Expanding on previous findings, we found that relative gain is not uniform across the population, but depends on the tuning preferences of the neurons (Fig. 3). The most salient effect is that neurons with a preference for high spatial frequencies have relative gains 25\% larger than those tuned for small spatial frequencies. A decoding analysis showed that, operating points.
A

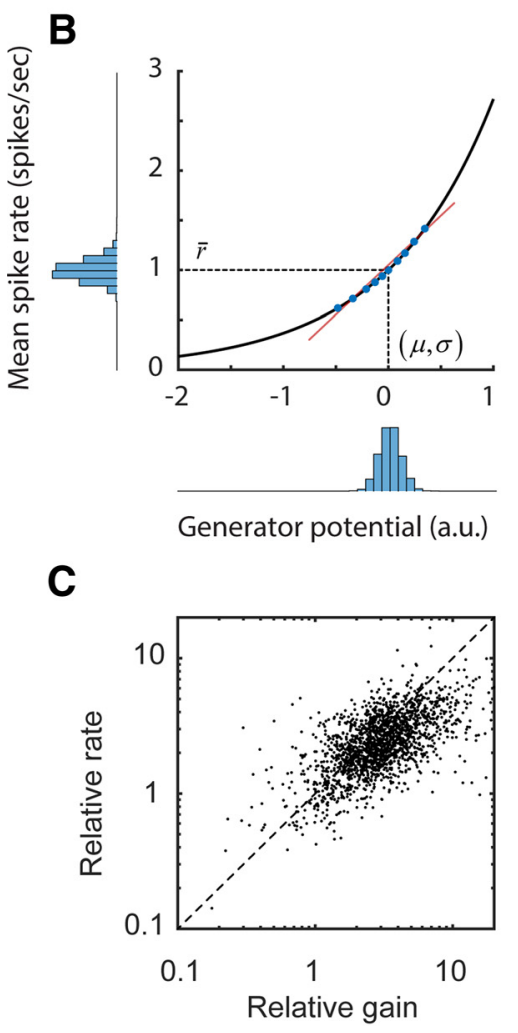

D

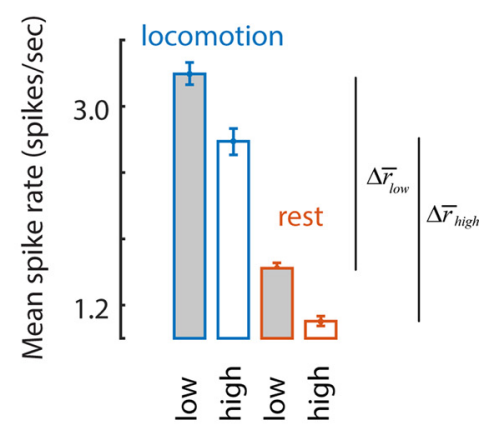

$\mathbf{E}$

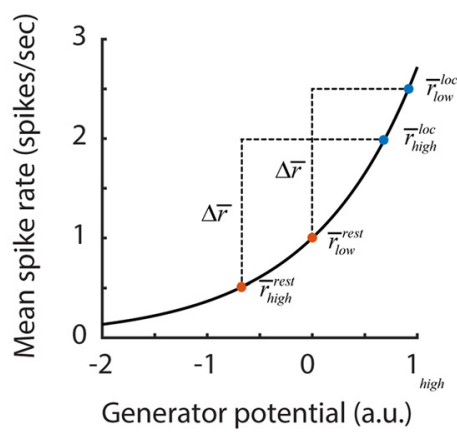

$\mathbf{F}$

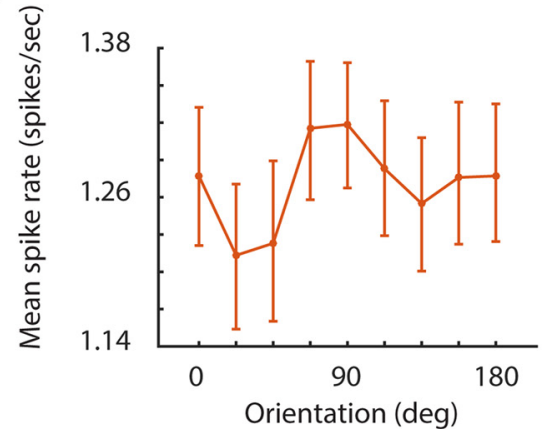

Figure 4. Changes in the operating point of neurons may explain differential gain changes. $\boldsymbol{A}, \mathrm{Scatter}$ plot of the mean rate and gain of cells during periods of rest and locomotion. The dashed line represents the best linear fit. $\boldsymbol{B}$, An exponential spiking nonlinearity with a Gaussian input has the property that the mean response is proportional to the gain, consistent with the data in A. Here, $\bar{r}$ represents the mean spike rate and $(\mu, \sigma)$ represent the mean and SD of the generator potential. The blue filled dots represent a small subset of data of the joint distribution of the generator potential and the mean spike rate as related by the nonlinearity. The red line represents the best linear fit to these points. The slope of this line is the gain. C, As predicted by this simple rectification model, relative changes in gain are closely related to relative changes in mean spike rate. $\boldsymbol{D}$, Cells with low- and high-spatial-frequency preferences have different operating points during rest and locomotion. The relative ordering of these rates, along with the approximate identity relationship shown in $\boldsymbol{C}$, explain how differential gain increases may occur. $\boldsymbol{E}$, Graphic summary of the relationship in $\boldsymbol{D}$ depicting the relative positioning of operating points of cells with low- and high-spatialfrequency preferences along the spiking nonlinearity. $\boldsymbol{F}$, There is a trend of mean rate with orientation that complements the dependence of relative gain with orientation (Fig. $3 B$ ), consistent with the proposed explanation based on the shifts of the

during locomotion, there is an enhanced visual representation that supports better discriminability of high-contrast stimuli, in particular stimuli at high spatial frequencies (Fig. 5).

Although arbitrary state-dependent changes in tuning could make the neural code ambiguous and difficult to read, a simple downstream decoder can read out cortical signals efficiently when only gain is modulated and receptive field tuning curves are approximately invariant. In this way, increased resources (neuronal action potentials and their corresponding metabolic cost) can be deployed during moments of high be- 


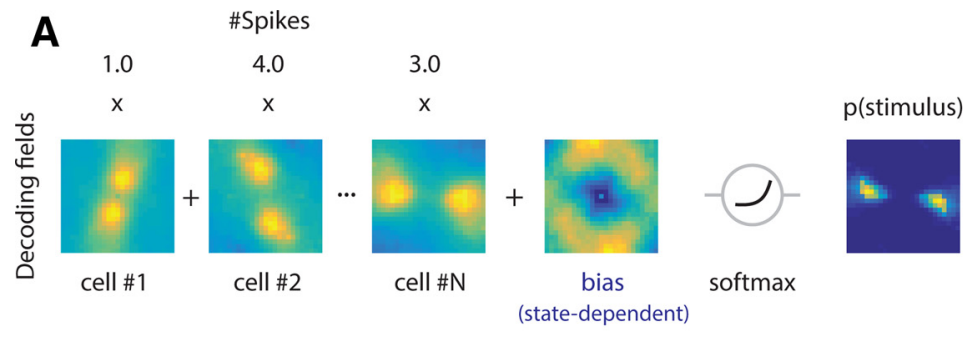

B

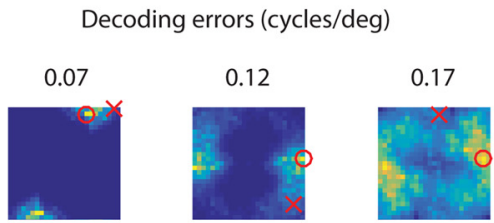

C

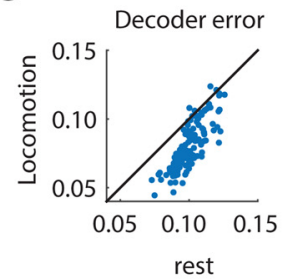

D

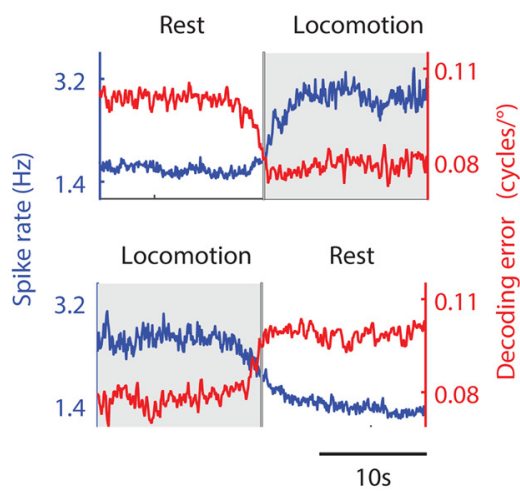

E

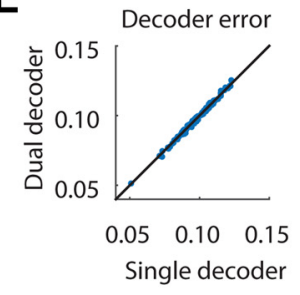

G

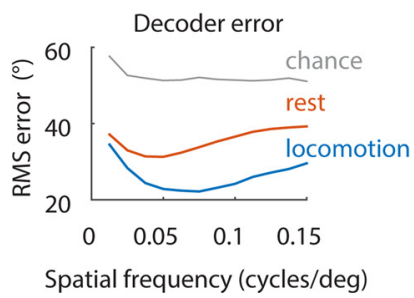

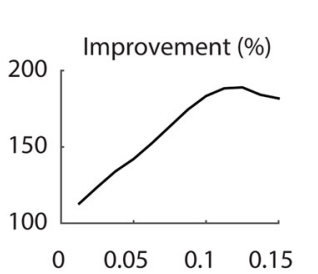

Spatial frequency (cycles/deg)

Figure 5. Locomotion enhances the population code and the cortical representation of high spatial frequencies. $\boldsymbol{A}$, The decoder consisted of the linear weighting of decoding fields by spike rates over the Fourier domain plus a state-dependent bias term, followed by a soft-max nonlinearity (Materials and Methods). The result is an estimate of the probability of the stimulus given the population firing rate, $p_{\text {(stimuluss }} \cdot \mathbf{B}$, Examples of $p_{\text {(stimulus) }}$ along with the estimated (red circle) and presented (red cross) stimuli together with the decoding error measured as the Euclidean distance between these two points on the Fourier plane. $\boldsymbol{C}$, Decoding error is smaller during locomotion compared with during rest. $\boldsymbol{D}$, Average changes in population spike rate and decoding error during transitions from rest to locomotion (top) and from locomotion to rest (bottom). $\boldsymbol{E}$, A single decoder that only modifies the bias in a state-dependent manner performs as well as two separate decoders for each state. In other words, there is no increase in performance if we allow the model to fit different Fourier kernels during locomotion and rest. $\boldsymbol{F}$, Decoding of orientation as a function of behavioral state and spatial frequency. Gray line represents chance performance. The lowest decoding errors are achieved during locomotion. $\mathbf{G}$, Relative improvement of decoder performance during locomotion versus rest. The relative improvement, in percentage, is defined as 100 * (RMS_loc - RMS_chance)/(RMS_rest RMS_chance). The relative improvement in decoding accuracy is concentrated at high spatial frequencies, where gain increases are more pronounced.

havioral demand such as locomotion, improving the representation of relevant stimuli (Lennie, 2003). Therefore, the neuronal code is shaped adaptively to increase spatial resolution in V1 without increasing the complexity or burden of decoding in downstream areas.

The preferential enhancement of high-spatial-frequency information lends additional support to the idea that changes in V1 during periods of increased alertness and locomotion in rodents resemble the changes that occur in nonhuman pri- mates during the allocation of spatial attention (Harris and Thiele, 2011). Specifically, the allocation of spatial attention in human subjects leads to increased spatial resolution, an effect that has been hypothesized to be driven by a selective increase in response gain at high spatial frequencies (AntonErxleben and Carrasco, 2013). We found that such an effect is evident in mouse V1, where differential gain modulation enhances the representation of high spatial frequencies during increased levels of alertness and locomotion. The evolutionary advantage of such enhancement is unclear. During locomotion, one expects the retinal image to become blurred by motion (Barlow and Olshausen, 2004). This might be particularly problematic in species that are unable to stabilize the retinal image by ocular tracking of external targets, such as mice. Perhaps the preferential enhancement of high spatial frequencies evolved as a way to counteract such loss of information during self-motion relative to a stationary environment.

Our findings indicate that the dependence of relative gain on the preferred tuning parameters of neurons is linked to corresponding differences in how their operating points shift across states. During rest, cells with a preference for high spatial frequency have smaller mean responses and gains than cells with a preference for low spatial frequency (Fig. 4). How this relationship arises remains unknown and we can only speculate at this point. One possibility is that the spatial distribution of the geniculate inputs required to generate a band-pass filter with a high-spatial-frequency preference involves the pooling of $\mathrm{ON}$ and OFF center cells to generate the $\mathrm{ON}$ and OFF subregions of simple cells. In contrast, cells with low-pass-spatial frequency profiles may be dominated by a fewer number of inputs from geniculate cells with the same center sign. One could conjecture that cells with balanced ON/OFF inputs and high-spatialfrequency selectivity are in a higher conductance resting state (and lower gain) than their counterparts with lowspatial-frequency selectivity. Therefore, the difference in the operating points may be a consequence of the number of synaptic inputs required to make up the corresponding receptive fields.

\section{References}

Adesnik H, Bruns W, Taniguchi H, Huang ZJ, Scanziani M (2012) A neural circuit for spatial summation in visual cortex. Nature 490:226-231. CrossRef Medline 
Ahrens MB, Paninski L, Sahani M (2008) Inferring input nonlinearities in neural encoding models. Network 19:35-67. CrossRef Medline

Anton-Erxleben K, Carrasco M (2013) Attentional enhancement of spatial resolution: linking behavioural and neurophysiological evidence. Nat Rev Neurosci 14:188-200. CrossRef Medline

Arroyo S, Bennett C, Hestrin S (2014) Nicotinic modulation of cortical circuits. Front Neural Circuits 8:30. CrossRef Medline

Ayaz A, Saleem AB, Schölvinck ML, Carandini M (2013) Locomotion controls spatial integration in mouse visual cortex. Curr Biol 23:890-894. CrossRef Medline

Barlow HB, Olshausen BA (2004) Convergent evidence for the visual analysis of optic flow through anisotropic attenuation of high spatial frequencies. J Vis 4:415-426. CrossRef Medline

Beatty J (1982) Task-evoked pupillary responses, processing load, and the structure of processing resources. Psychol Bull 91:276-292. CrossRef Medline

Bennett C, Arroyo S, Hestrin S (2013) Subthreshold mechanisms underlying state-dependent modulation of visual responses. Neuron 80:350-357. CrossRef Medline

Bereshpolova Y, Stoelzel CR, Zhuang J, Amitai Y, Alonso JM, Swadlow HA (2011) Getting drowsy? Alert/nonalert transitions and visual thalamocortical network dynamics. J Neurosci 31:17480-17487. CrossRef Medline

Boyd S, Vandenberghe L (2004) Convex optimization. Cambridge: Cambridge University.

Carrasco M, Barbot A (2014) How attention affects spatial resolution. Cold Spring Harb Symp Quant Biol 79:149-160. CrossRef Medline

Carrasco M, Yeshurun Y (2009) Covert attention effects on spatial resolution. Prog Brain Res 176:65-86. CrossRef Medline

Carrasco M, Williams PE, Yeshurun Y (2002) Covert attention increases spatial resolution with or without masks: support for signal enhancement. J Vis 2:467-479. Medline

Chen TW, Wardill TJ, Sun Y, Pulver SR, Renninger SL, Baohan A, Schreiter ER, Kerr RA, Orger MB, Jayaraman V, Looger LL, Svoboda K, Kim DS (2013) Ultrasensitive fluorescent proteins for imaging neuronal activity. Nature 499:295-300. CrossRef Medline

Deneve S, Latham PE, Pouget A (1999) Reading population codes: a neural implementation of ideal observers. Nat Neurosci 2:740-745. CrossRef Medline

Erisken S, Vaiceliunaite A, Jurjut O, Fiorini M, Katzner S, Busse L (2014) Effects of locomotion extend throughout the mouse early visual system. Curr Biol 24:2899-2907. CrossRef Medline

Fu Y, Tucciarone JM, Espinosa JS, Sheng N, Darcy DP, Nicoll RA, Huang ZJ, Stryker MP (2014) A cortical circuit for gain control by behavioral state. Cell 156:1139-1152. CrossRef Medline

Goard M, Dan Y (2009) Basal forebrain activation enhances cortical coding of natural scenes. Nat Neurosci 12:1444-1449. CrossRef Medline

Golla H, Ignashchenkova A, Haarmeier T, Thier P (2004) Improvement of visual acuity by spatial cueing: a comparative study in human and nonhuman primates. Vision Res 44:1589-1600. CrossRef Medline

Graf AB, Kohn A, Jazayeri M, Movshon JA (2011) Decoding the activity of neuronal populations in macaque primary visual cortex. Nat Neurosci 14:239-245. CrossRef Medline

Granit R (1955) Receptors and sensory perception: a discussion of aims, means, and results of electrophysiological research into the process of reception. New Haven, CT: Yale University.

Greenberg DS, Kerr JN (2009) Automated correction of fast motion artifacts for two-photon imaging of awake animals. J Neurosci Methods 176: 1-15. CrossRef Medline

Harris KD, Thiele A (2011) Cortical state and attention. Nat Rev Neurosci 12:509-523. CrossRef Medline

Jazayeri M, Movshon JA (2006) Optimal representation of sensory information by neural populations. Nat Neurosci 9:690-696. CrossRef Medline

Johnstone IM, Silverman BW (1997) Wavelet threshold estimators for data with correlated noise. J Royal Stat Soc B 59:319-351. CrossRef
Keller GB, Bonhoeffer T, Hübener M (2012) Sensorimotor mismatch signals in primary visual cortex of the behaving mouse. Neuron 74:809-815. CrossRef Medline

Lee SH, Dan Y (2012) Neuromodulation of brain states. Neuron 76: 209-222. CrossRef Medline

Lennie P (2003) The cost of cortical computation. Curr Biol 13:493-497. CrossRef Medline

Malone BJ, Ringach DL (2008) Dynamics of tuning in the Fourier domain. J Neurophysiol 100:239-248. CrossRef Medline

McCullagh P, Nelder JA (1989) Generalized linear models. Boca Raton, FL: CRC.

Murphy PR, O'Connell RG, O'Sullivan M, Robertson IH, Balsters JH (2014) Pupil diameter covaries with BOLD activity in human locus coeruleus. Hum Brain Mapp 35:4140-4154. CrossRef Medline

Niell CM, Stryker MP (2010) Modulation of visual responses by behavioral state in mouse visual cortex. Neuron 65:472-479. CrossRef Medline

Nykamp DQ, Ringach DL (2002) Full identification of a linear-nonlinear system via cross-correlation analysis. J Vis 2:1-11. Medline

Ozden I, Lee HM, Sullivan MR, Wang SS (2008) Identification and clustering of event patterns from in vivo multiphoton optical recordings of neuronal ensembles. J Neurophysiol 100:495-503. CrossRef Medline

Pnevmatikakis EA, Gao Y, Soudry D, Pfau D, Lacefield C, Poskanzer K, Bruno R, Yuste R, Paninski L (2014) A structured matrix factorization framework for large scale calcium imaging data analysis. arXiv preprint arXiv: 14092903.

Polack PO, Friedman J, Golshani P (2013) Cellular mechanisms of brain state-dependent gain modulation in visual cortex. Nat Neurosci 16: 1331-1339. CrossRef Medline

Poulet JF, Petersen CC (2008) Internal brain state regulates membrane potential synchrony in barrel cortex of behaving mice. Nature 454:881-885. CrossRef Medline

Reimer J, Froudarakis E, Cadwell CR, Yatsenko D, Denfield GH, Tolias AS (2014) Pupil fluctuations track fast switching of cortical states during quiet wakefulness. Neuron 84:355-362. CrossRef Medline

Reynolds JH, Heeger DJ (2009) The normalization model of attention. Neuron 61:168-185. CrossRef Medline

Ringach DL, Malone BJ (2007) The operating point of the cortex: neurons as large deviation detectors. J Neurosci 27:7673-7683. CrossRef Medline

Ringach DL, Sapiro G, Shapley R (1997) A subspace reverse-correlation technique for the study of visual neurons. Vision Res 37:2455-2464. CrossRef Medline

Saleem AB, Ayaz A, Jeffery KJ, Harris KD, Carandini M (2013) Integration of visual motion and locomotion in mouse visual cortex. Nat Neurosci 16:1864-1869. CrossRef Medline

Sara SJ (2009) The locus coeruleus and noradrenergic modulation of cognition. Nat Rev Neurosci 10:211-223. CrossRef Medline

Shuler MG, Bear MF (2006) Reward timing in the primary visual cortex. Science 311:1606-1609. CrossRef Medline

Smith SL, Häusser M (2010) Parallel processing of visual space by neighboring neurons in mouse visual cortex. Nat Neurosci 13:1144-1149. CrossRef Medline

Tan AY, Chen Y, Scholl B, Seidemann E, Priebe NJ (2014) Sensory stimulation shifts visual cortex from synchronous to asynchronous states. Nature 509:226-229. CrossRef Medline

Vogelstein JT, Packer AM, Machado TA, Sippy T, Babadi B, Yuste R, Paninski L (2010) Fast nonnegative deconvolution for spike train inference from population calcium imaging. J Neurophysiol 104:3691-3704. CrossRef Medline

Wu MC, David SV, Gallant JL (2006) Complete functional characterization of sensory neurons by system identification. Annu Rev Neurosci 29: 477-505. CrossRef Medline

Zhuang J, Bereshpolova Y, Stoelzel CR, Huff JM, Hei X, Alonso JM, Swadlow HA (2014) Brain state effects on layer 4 of the awake visual cortex. J Neurosci 34:3888-3900. CrossRef Medline 